\title{
Origin of the anomalous low-temperature phase transition in $\mathrm{BaVS}_{3}$
}

\author{
R. A. de Souza, ${ }^{1}$ U. Staub, ${ }^{1}$ V. Scagnoli, ${ }^{1}$ M. Garganourakis,,${ }^{1}$ Y. Bodenthin,,${ }^{1}$ and H. Berger ${ }^{2}$ \\ ${ }^{1}$ Swiss Light Source, Paul Scherrer Institut, CH-5232 Villigen PSI, Switzerland \\ ${ }^{2}$ Institut de Physique des Nanostructures, Ecole Polytechnique Fédérale (EPFL), CH-1015 Lausanne, Switzerland
}

(Received 22 March 2011; published 21 July 2011)

\begin{abstract}
The origin of the anomalous electronic phase transition $\left(T_{X} \approx 30 \mathrm{~K}\right)$ caused by a single-electron-occupied $t_{2 g}$ orbital in quasi-one-dimensional $\mathrm{BaVS}_{3}$ is studied by means of resonant soft x-ray diffraction taken at the V $L_{2,3}$ absorption edges. The energy and azimuthal angle dependence of the observed $(0 k l)$ reflection can be explained by the presence of at least two electronically different $\mathrm{V}$ contributions. Moreover, analysis of the scattered $\mathrm{x}$-ray polarization indicates that the observed reflection is purely magnetic in origin, which discourages models with orbital and charge modifications occurring at $T_{X}$. The dependence of the reflection on left or right circular polarized incoming $\mathrm{x}$ rays indicates that the incommensurability of the reflection is connected to a helical component of the magnetic structure. These results, together with those from neutron scattering, are discussed in terms of the different models proposed for the electronic and magnetic ground state.
\end{abstract}

DOI: 10.1103/PhysRevB.84.014409

PACS number(s): 75.25.-j, 78.70.Ck

\section{INTRODUCTION}

Transition metal oxides exhibiting strong electron correlations present phenomena like, metal-insulator (MI) transition, charge, spin and orbital ordering, and superconductivity. ${ }^{1}$ In contrast to oxides, sulfides are much less explored. They present reduced ionicity of the cation-anion bond compared to oxides, thereby increasing the effect of hybridization. The compound $\mathrm{BaVS}_{3}$ crystallizes in a hexagonal $(\mathrm{H})$ structure $\left(P 6_{3} / m m c\right)$ with two formula units per unit cell and the formation of $\mathrm{VS}_{6}$-octahedra chains running along the $c$ axis. In this phase, the distorted $\left(D_{3 d}\right)$ sulfur octahedral environment of vanadium leads to a crystal field splitting of the V $(3 d)$ orbitals into a higher energy $A_{1 g}$ level and two quasidegenerate $E_{g}$ states, with only one electron to share between these orbitals. The V-V distance in the chain $(2.84 \AA)$ is much shorter than the $\mathrm{V}-\mathrm{V}$ interchain distance $(6.72 \AA)$, which gives quasi-one-dimensional structural properties to this compound. However, the anisotropy of the conductivity measured along and perpendicular to the chain direction happens to be quite low. ${ }^{2}$ This compound undergoes a structural transition at $240 \mathrm{~K}$ to an orthorhombic (O) structure $\left(C m c 2_{1}\right),{ }^{3}$ lifting the degeneracy of the $E_{g}$ states. An MI transition is observed at $T_{\mathrm{MI}}=70 \mathrm{~K},{ }^{4}$ below which a charge density wave with superstructure corresponding to a doubling along the chain direction develops. ${ }^{5,6}$ This results in a monoclinic (M) structure ( Im) deformation, with four inequivalent $\mathrm{V}$ atoms per unit. Electronic structure calculations indicate a departure from the balanced occupation of $A_{1 g}$ and $E_{g}$ states in the monoclinic phase, toward an increase in the $E_{g}$ filling. ${ }^{7}$ Moreover, at $T_{\mathrm{MI}}$ the magnetic susceptibility show a maximum, ${ }^{2}$ although no long-range magnetic order has been detected. An additional transition is observed at $T_{X}=30 \mathrm{~K}$, whose origin is still a matter of controversy. At this temperature an anomaly is observed in the magnetic susceptibility. ${ }^{2}$ Neutron powder diffraction studies provide evidence of superlattice (SL) reflections below $T_{X} .{ }^{8}$ Based on the assumption of no interchain coupling, the modulation vector of $(0.2260 .2260)_{H}$ of a possible magnetic order was suggested. NMR studies suggest that orbital order occurs at this temperature. ${ }^{9}$ Other studies have proposed the coexistence of orbital and magnetic order. ${ }^{10}$
Moreover, a review of the structural properties has also shown that below $40 \mathrm{~K}$ the structure is still monoclinic, but it changes slightly from its structure below the MI transition. ${ }^{11}$ Based on the bond valence sum method, the same structural study suggests that the four $\mathrm{V}$ sites present at this temperature would have different charges, indicative of charge order at the $\mathrm{V}$ ions. However, resonant X-ray diffraction (XRD) at the V K edge ${ }^{12}$ could be explained without charge ordering. The same XRD study proposes the existence of two charge-density-wave modulations that would cancel each other, leading to exclusive orbital ordering. Despite the many scenarios proposed, neither the exact nature of the transition at $T_{X}$ nor the exact electronic ground state is understood.

Resonant soft XRD is able to directly measure and disentangle charge, orbital, and magnetic ordering contributions in such a $3 d^{1}$ system by directly accessing the $3 d$ states responsible for physical properties. ${ }^{13,14}$ To clarify the origin of the $T_{X}$ transition observed in $\mathrm{BaVS}_{3}$ we have studied the associated SL reflection and determined its $\mathbf{q}$ position. We have also determined the temperature, energy, and polarization dependence of the SL reflections observed at $\mathrm{V} L_{2,3}$ edges. This indicates that the two SL reflections observed are both of magnetic origin. A simplified magnetic structure is proposed based on these results.

\section{EXPERIMENT}

Single crystals of $\mathrm{BaVS}_{3}$ were grown by the tellurium flux method, described elsewhere. ${ }^{15}$ Crystals obtained from the flux by sublimation have a needle-like shape with typical dimensions of $3 \times 1 \times 1 \mathrm{~mm}^{3}$. The crystal was precharacterized with $\mathrm{CuK} \alpha$ radiation at room temperature and with $2-\mathrm{keV} \mathrm{x}$ rays at $10 \mathrm{~K}$. The sample was aligned with the $\left[\begin{array}{lll}1 & 1 & 0\end{array}\right]_{H}$ direction normal to the surface, as this is the proposed direction of the SL structure. ${ }^{8}$ The sample was cooled to temperatures between 10 and $50 \mathrm{~K}$ using a helium-flow cryostat.

Resonant soft XRD experiments were performed at the RESOXS end station at the SIM beamline of the Swiss Light Source at the Paul Scherrer Institut. Measurements were carried out in a horizontal scattering geometry at the $\mathrm{V} L_{2,3}$ 
edges. The linear polarization of the incident radiation was either horizontal ( $\pi$; parallel to the scattering plane) or vertical ( $\sigma$; perpendicular to the scattering plane). Polarization analysis of the scattered radiation was performed using a graded $\mathrm{W} / \mathrm{C}$ multilayer setup. ${ }^{16,17}$ Rotations around the Bragg wave vector (azimuthal angle $\psi$ ) were achieved via a rotatable sample transfer fork with an accuracy of better than $5^{\circ}$. The origin of the azimuthal angle $\left(\psi=0^{\circ}\right)$ corresponds to the $\left[\begin{array}{lll}0 & 0 & 1\end{array}\right]_{H}$ direction lying within the horizontal scattering plane. The experiment was performed with either a focused $(50 \times 100 \mu \mathrm{m})$ or an unfocused $(2 \times 2 \mathrm{~mm})$ beam. In the latter case, the illuminated sample area was reduced using a mask of aluminized tape with a $1-\mathrm{mm}$-diameter aperture.

\section{RESULTS AND DISCUSSION}

Two SL reflections were observed at the $\mathrm{V} L_{3}$-edge resonance, at $q \cong 0.43 \AA^{-1}$ below $T_{X}$ and around $\left[\begin{array}{lll}1 & 1 & 0\end{array}\right]_{H}$, which are shown in Fig. 1 (labeled $\mathbf{A}$ and $\mathbf{B}$ ). These reflections can be indexed in the high-temperature hexagonal cell as $\mathrm{A},(0.2320 .232-0.016)_{H}$, and $\mathrm{B},(0.2320 .232+0.016)_{H}$. The incommensurability along $c$, thought to be small, is not negligible, since the two reflections are very clearly distinguished and symmetrically separated from the $\left[\begin{array}{lll}1 & 1 & 0\end{array}\right]_{H}$ direction. The horizontal, vertical, and out-of-page directions in Fig. 1 correspond to the $\left[\begin{array}{lll}-1 & 1 & 0\end{array}\right]_{H},\left[\begin{array}{lll}0 & 0 & 1\end{array}\right]_{H}$, and $\left[\begin{array}{lll}1 & 1 & 0\end{array}\right]_{H}$ orientations, respectively. The broad outspread of the peak in the direction of $\theta$ is due to the mosaicity of the crystal. This was confirmed by also measuring lattice reflections at a higher energy $(2 \mathrm{keV})$. The rocking curves of the lattice and SL reflections are shown in Fig. 2, and each presents a multipeak structure. The greater width of the SL peaks can be attributed to the shorter correlation length of the superstructure and absorption effects at the resonance, which decrease the penetration depth of the $\mathrm{x}$ rays from $1.5 \mu \mathrm{m}$ at $2 \mathrm{keV}$ to $0.2 \mu \mathrm{m}$ at resonance.

The $\left(\begin{array}{lll}1 & 1 & 0\end{array}\right)_{H}$ and its equivalent reflections in the hexagonal structure correspond to two inequivalent directions in the lowtemperature monoclinic cell: $\left[\begin{array}{lll}0 & 2 & 0\end{array}\right]_{M}$ and $\left[\begin{array}{lll}3 & 1 & 0\end{array}\right]_{M}$. Moreover,

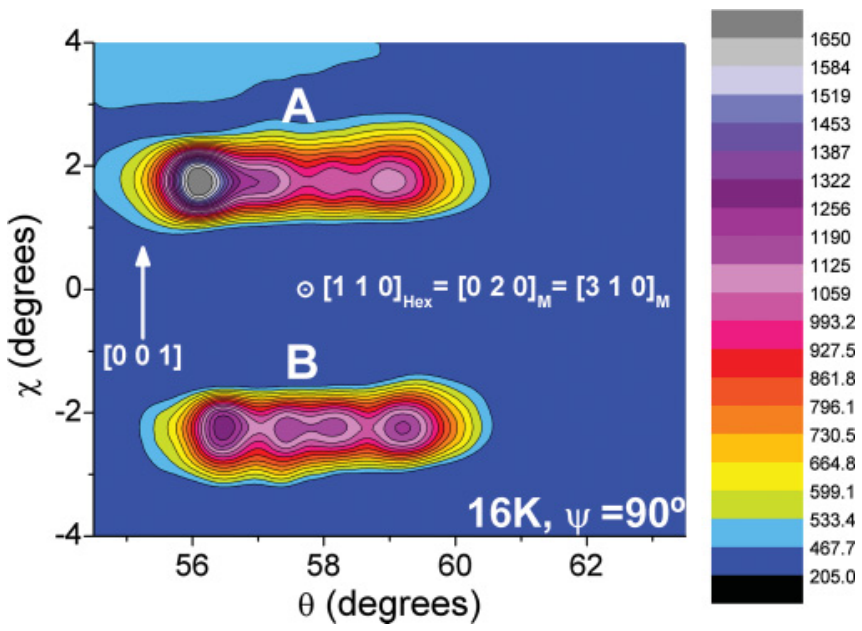

FIG. 1. (Color online) The $\mathrm{x}$-ray intensity maps around the $\left[\begin{array}{lll}1 & 1 & 0\end{array}\right]_{H}$ direction measured at resonance, at $16 \mathrm{~K}$ and $\psi=90^{\circ}$. The $\left[\begin{array}{lll}0 & 0 & 1\end{array}\right]_{H}$ direction is indicated.

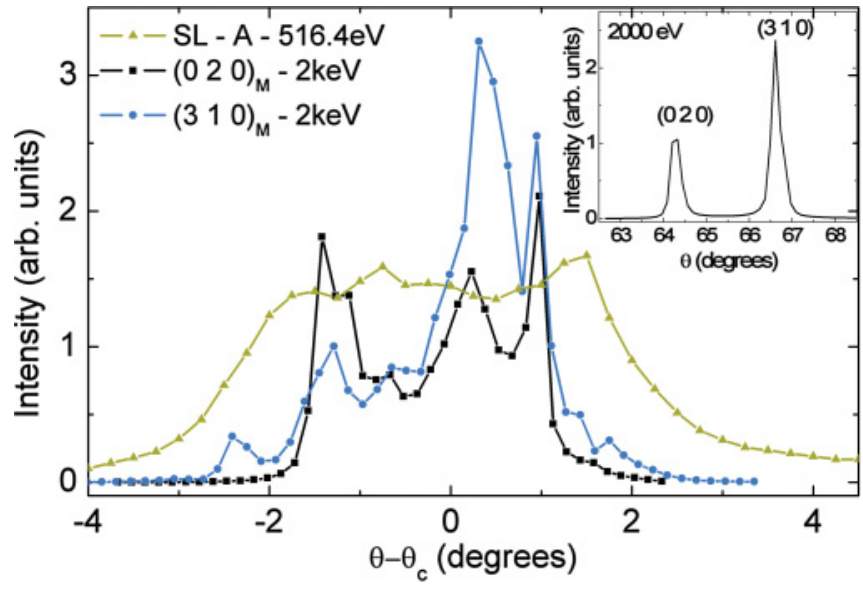

FIG. 2. (Color online) Rocking curves of the SL reflection A, taken at the $\mathrm{V} L_{3}$ edge, and rocking curves of the low-temperature monoclinic reflections $\left(\begin{array}{llll}0 & 2 & 0\end{array}\right)_{M}$ and $\left(\begin{array}{lll}3 & 1 & 0\end{array}\right)_{M}$, taken with $2-\mathrm{keV}$ $\mathrm{x}$ rays, at the same lateral position on the sample and incident/exit angles with a focused x-ray beam. Inset: The split to the $\left(\begin{array}{lll}1 & 1 & 0\end{array}\right)_{H}$ reflection at a low temperature $(10 \mathrm{~K})$.

the hexagonal $\left(\begin{array}{lll}1 & 1 & 0\end{array}\right)_{H}$ reflection is split at low temperatures, as demonstrated in the inset in Fig. 2, where both $\left(\begin{array}{lll}0 & 2 & 0\end{array}\right)_{M}$ and (3 10$)_{M}$ reflections are observed. There are, correspondingly, two main possibilities to index the SL reflection in monoclinic symmetry. Limiting the incommensurability to the minimal number of crystal axes results in the monoclinic cell in $\mathbf{A}$ being

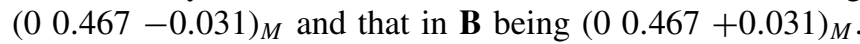
Note that the $c$ axis of the monoclinic cell is about twice that of the hexagonal cell. The temperature dependence of reflection A is presented in Fig. 3; the peak vanishes at $\approx 32 \mathrm{~K}$, consistent with the $T_{X}$ transition. Both reflections, $\mathbf{A}$ and $\mathbf{B}$, present the same temperature behavior (only one is shown).

The energy dependence of SL reflection A measured with $\pi$ and $\sigma$ incident light is presented in Fig. 4 for two values of the azimuthal angle. Spectra measured with different incoming $\mathrm{X}$-ray polarization show very different energy dependencies. In addition, the relative intensity of the features observed in the energy dependence are significantly different between the

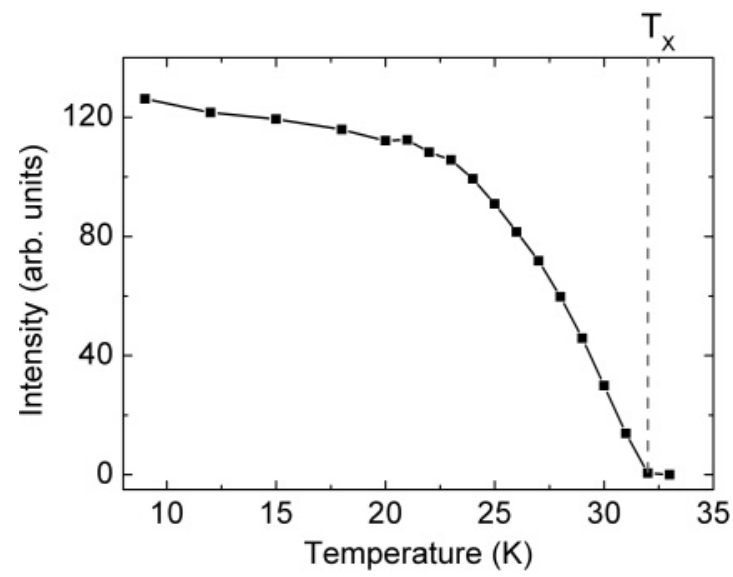

FIG. 3. Temperature dependence of SL reflection $\mathbf{A}$ of $\mathrm{BaVS}_{3}$ taken with $\pi$ incident radiation at $516.4 \mathrm{eV}$. The vertical dashed line identifies $T_{X}$ as being approximately $32 \mathrm{~K}$. 


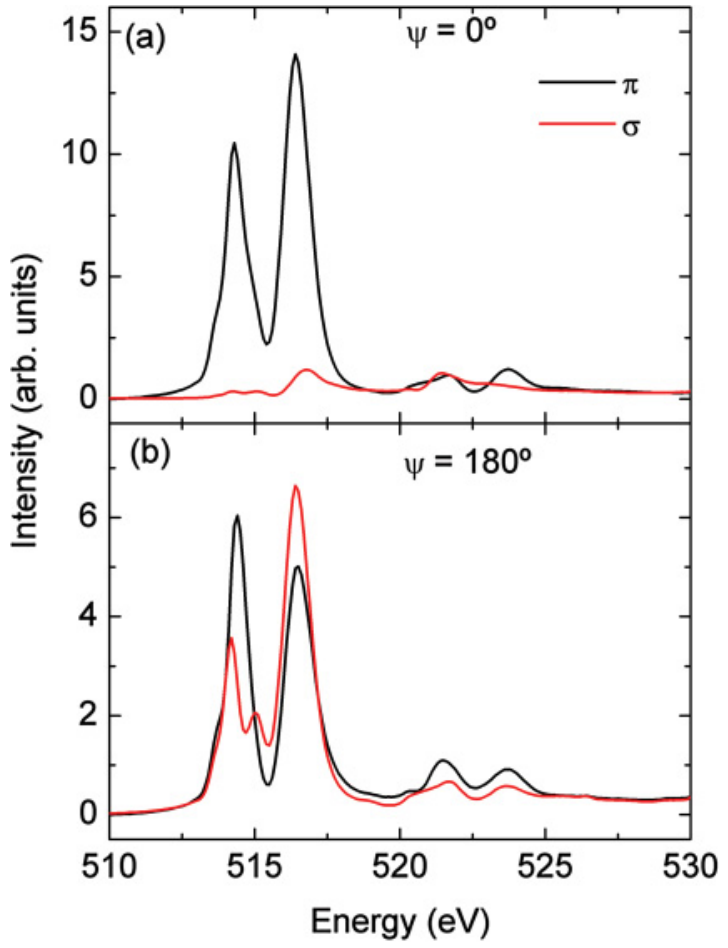

FIG. 4. (Color online) Energy dependence of the SL reflection A measured at $16 \mathrm{~K}$ with $\pi$ and $\sigma$ incident polarization of $\mathrm{x}$ rays at two azimuthal angles: (a) $\psi=0^{\circ}$ and (b) $\psi=180^{\circ}$.

two different azimuthal angles. A single electronic component would lead only to a variation of the overall intensity when changing the azimuthal angle or the incident $\mathrm{x}$-ray polarization. This is not what we observe. The variation indicates that more than one electronic component contributes to the signal. The energy dependence observed for peak $\mathbf{B}$ shows the same shape as observed for peak $\mathbf{A}$, at an azimuth shifted by $\pi$, which means that $I_{\mathbf{A}}^{\psi}(E)=I_{\mathbf{B}}^{\psi+\pi}(E)$. This relation indicates that the two incommensurate reflections, $\mathbf{A}$ and $\mathbf{B}$, represent the same physics and represent simply two different domains.

The energy dependence of the SL reflection also depends on the helicity of the incoming circularly polarized light, as shown in Fig. 5. The differences in the shape of the spectra for right and left circularly polarized light are evidence of a helical component of the magnetic structure or, possibly, additional multipolar contributions as observed for $\mathrm{CuO}^{18}$

Polarization analysis of the scattered beam gives further information on the origin of the measured signal. For a detailed description of the polarization dependence of resonant diffraction, we refer to Hill and McMorrow. ${ }^{19}$ For charge scattering, one expects intensity only in the unrotated channels: $\pi-\pi^{\prime}$ and $\sigma-\sigma^{\prime}$. For magnetic scattering, on the other hand, the $\sigma-\sigma^{\prime}$ channel is absent and the channels of the rotated light, $\sigma-\pi^{\prime}$ and $\pi-\sigma^{\prime}$, have an opposite complex phase, which depends on the scattering angle, and have opposite sign. The situation for orbital scattering is more complex and all four polarization channels may be allowed and may depend on the azimuth.

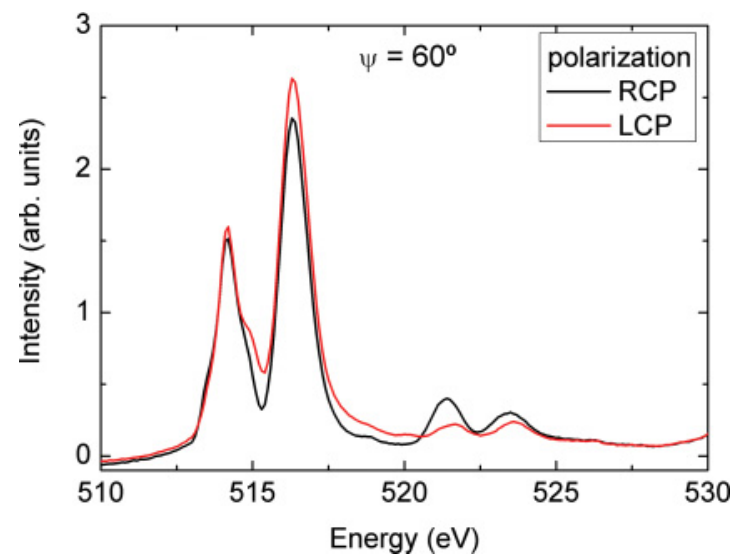

FIG. 5. (Color online) Energy dependence of the SL reflection A with right (RCP) and left (LCP) circular polarized light, collected at $20 \mathrm{~K}$.

Polarization analysis has been performed for $\psi=0^{\circ}$, determining all four inequivalent polarization contributions. The corresponding rocking curves are shown in Fig. 6. No signal is present in the $\sigma-\sigma^{\prime}$ channel. Intensity is observed in the other three channels: $I_{\pi \pi^{\prime}} \neq I_{\pi \sigma^{\prime}} \neq I_{\sigma \pi^{\prime}} \neq 0$. This directly excludes charge ordering for the $T_{X}$ transition and strongly supports the magnetic origin of the signal, which discourages models with orbital ordering occurring at $T_{X} \cdot{ }^{12,20}$

For an electric dipole transition, the resonant magnetic scattering amplitude is given by ${ }^{19,21}$

$$
f_{\epsilon^{\prime} \epsilon}^{\mathrm{XRES}}=-\frac{3}{4 k} i\left(\epsilon^{\prime} \times \epsilon\right) \cdot \mathbf{z}_{j}\left[F_{11}-F_{1-1}\right],
$$

where $\epsilon$ and $\epsilon^{\prime}$ are the polarization of the incident and scattered radiation, $\mathbf{z}_{j}$ is the quantization of the magnetic moment

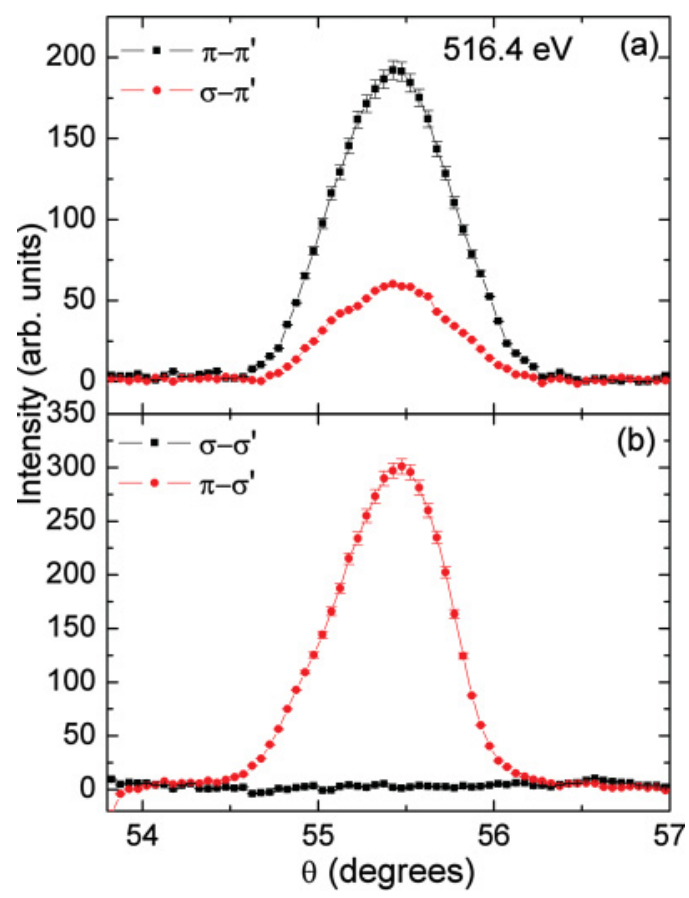

FIG. 6. (Color online) Polarization dependence of SL reflection A taken at $516.4 \mathrm{eV}$ in the four polarization channels. 


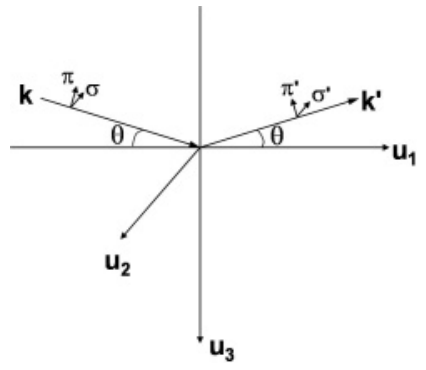

FIG. 7. Coordinate system used for calculation of the polarization-dependent resonant cross section.

at atom $j$, and $F_{11}$ and $F_{1-1}$ are atomic properties of the initial and excited states of the $\mathrm{V}$ ions. These are related to the $3 d$ magnetic moment and the overlap integral. The coordinate system defined with respect to the diffraction plane is represented in Fig. 7. $\pi$ and $\sigma$ represent the electric field direction of the $\mathrm{x}$-ray polarization parallel and perpendicular to the scattering plane, respectively. $\mathbf{q}=\mathbf{k}-\mathbf{k}^{\prime}$ is the wavevector transfer, and $\mathbf{k}$ and $\mathbf{k}^{\prime}$ are the wave vectors of the incident and diffracted radiation, respectively. The sum over magnetic ions $j$ gives the magnetic structure factor

$$
M_{\epsilon^{\prime} \epsilon}^{\mathrm{XRS}}=\sum_{j} \exp \left(i \mathbf{q} \cdot \mathbf{r}_{j}\right) f_{\epsilon^{\prime} \epsilon}^{\mathrm{XRES}} .
$$

Without polarization analysis, the diffracted intensity $I_{\epsilon}^{\text {XRES }}$ for the different incident polarization is given by

$$
\begin{gathered}
I_{\sigma}^{\mathrm{XRES}}=\left|M_{\sigma^{\prime} \sigma}\right|^{2}+\left|M_{\pi^{\prime} \sigma}\right|^{2}, \\
I_{\pi}^{\mathrm{XRES}}=\left|M_{\sigma^{\prime} \pi}\right|^{2}+\left|M_{\pi^{\prime} \pi}\right|^{2}, \\
I_{\chi}^{\mathrm{XRES}}=\frac{1}{2}\left(\left|M_{\sigma^{\prime} \sigma}\right|^{2}+\left|M_{\pi^{\prime} \sigma}\right|^{2}+\left|M_{\sigma^{\prime} \pi}\right|^{2}+\left|M_{\pi^{\prime} \pi}\right|^{2}\right) \\
+\chi \operatorname{Im}\left[M_{\sigma^{\prime} \sigma} M_{\sigma^{\prime} \pi}^{*}+M_{\pi^{\prime} \sigma} M_{\pi^{\prime} \pi}^{*}\right],
\end{gathered}
$$

where $\chi=+1$ indicates the right circular polarized and $\chi=$ -1 represents the left circular polarized incident beam.

The azimuthal angle dependence of the intensity ratio $\frac{I^{+}-I^{-}}{I^{+}+I^{-}}$, where the + and - signs refer to the sign of $\chi$, measured for reflection $\mathbf{A}$ at the $L_{2}$ edge $(521.4 \mathrm{eV})$, is presented in Fig. 8 . The differences in intensity measured with circular light of

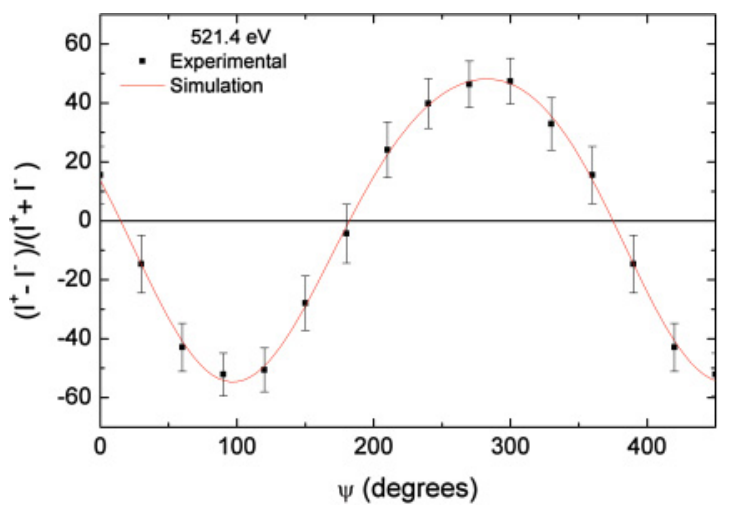

FIG. 8. (Color online) Azimuthal angle $(\psi)$ dependence of the intensity ratio $\frac{I^{+}-I^{-}}{I^{+}+I^{-}}$of reflection A measured at the main feature of $L_{2}(521.4 \mathrm{eV})$. Symbols are experimental points, and the solid line is a fit. different helicities are greater at this feature of the $L_{2}$ edge, as shown in Fig. 5. This ratio, also called the figure of merit, is more precise than the azimuthal angle dependence measured with only one polarization, since it corrects for systematic experimental errors due to the elongated shape of the peaks.

In terms of the linear polarized radiation, the figure of merit of circular light is given by

$$
\frac{I^{+}-I^{-}}{I^{+}+I^{-}}=\frac{2 \operatorname{Im}\left(M_{\sigma^{\prime} \sigma} M_{\sigma^{\prime} \pi}^{*}+M_{\pi^{\prime} \sigma} M_{\pi^{\prime} \pi}^{*}\right)}{\left|M_{\sigma^{\prime} \sigma}\right|^{2}+\left|M_{\pi^{\prime} \sigma}\right|^{2}+\left|M_{\sigma^{\prime} \pi}\right|^{2}+\left|M_{\pi^{\prime} \pi}\right|^{2}} .
$$

In Fig. 8 one can see that for $\psi \approx 0$ the intensity ratio is 0 . Assuming a single magnetic moment rotating for consecutive atoms, $\frac{I^{+}-I^{-}}{I^{+}+I^{-}}(\psi=0)=0$ is only possible for a magnetic spiral in the $u_{1} u_{3}$ plane, which for $\psi=0$ corresponds to the bc plane of the monoclinic structure. In this case the moment direction at $\psi=0$ can be expressed as $z_{j}^{0}=\mathbf{u}_{\mathbf{1}} \cos \left(\tau \cdot \mathbf{r}_{\mathbf{j}}\right)+\mathbf{u}_{\mathbf{3}} \sin \left(\tau \cdot \mathbf{r}_{\mathbf{j}}\right)$. To calculate the azimuthal angle $(\psi)$ dependence, one needs $z_{j}(\psi)$, given by

$$
z_{j}(\psi)=\left(\begin{array}{ccc}
\cos (\psi) & \sin (\psi) & 0 \\
-\sin (\psi) & \cos (\psi) & 0 \\
0 & 0 & 1
\end{array}\right) z_{j}^{0} .
$$

The cross terms of polarization in Eq. (1) give $\sigma^{\prime} \times \sigma=$ $0, \sigma^{\prime} \times \pi=\mathbf{k}, \pi^{\prime} \times \sigma=-\mathbf{k}^{\prime}$, and $\pi^{\prime} \times \pi=\mathbf{k}^{\prime} \times \mathbf{k}$. The incident and diffracted wave vectors can be expressed as $\mathbf{k}=$ $\widehat{u}_{1} \cos (\theta)+\widehat{u}_{3} \sin (\theta)$ and $\mathbf{k}^{\prime}=\widehat{u}_{1} \cos (\theta)-\widehat{u}_{3} \sin (\theta)$. Using these terms and the expression of $z_{j}(\psi)$ [Eq. (7)] in Eq. (2), we obtain

$$
\begin{aligned}
& \frac{I^{+}-I^{-}}{I^{+}+I^{-}}(\psi) \\
& \propto \frac{\sin (\theta) \sin (2 \theta) \sin (\psi)}{2 \sin ^{2}(\theta)+2 \cos ^{2}(\theta) \cos ^{2}(\psi)+\sin ^{2}(2 \theta) \sin ^{2}(\psi)} .
\end{aligned}
$$

The data measured at the $L_{2}$ edge have been fitted with this model, which closely reproduces the azimuthal evolution of $\frac{I^{+}-I^{-}}{I^{+}+I^{-}}(\psi)$, as shown in Fig. 8. This means that we have a magnetic spiral component with moments lying in the bc plane contributing to the structure factor. This does not necessarily mean that there is no component of the magnetic moment along the $a$ axis. However, such components will not have a helical component.

We can now compare the exact index of the reflections observed here to those observed by neutron powder diffraction. ${ }^{8}$ In that study, three magnetic reflections were observed. The first reflection, at $q=0.425 \AA^{-1}$, corresponds to the two reflections observed here. Writing the index with respect to the commensurate reflection $\left(0 \frac{1}{2} 0\right)$, we have $\left(0 \frac{1}{2}-\delta_{k} \pm \delta_{l}\right)_{M}$, with $\delta_{k}=0.033$ and $\delta_{l}=0.031$. The second reflection, at $q=0.74 \AA^{-1}$, can be indexed as $\left(1 \frac{1}{2}+\delta_{k} \pm \delta_{l}\right)_{M}$. Finally, the third reflection, at $q=1.17 \AA^{-1}$, can be indexed as $\left(0 \frac{1}{2}-\right.$ $\left.\delta_{k} 2-\delta_{l}\right)_{M}$. All these reflection are represented in Fig. 9 . The reflections $\left(1 \frac{1}{2}+\delta_{k} 2 \pm \delta_{l}\right)_{M}$ and $\left(0 \frac{1}{2}-\delta_{k} 2+\delta_{l}\right)_{M}$ are additionally represented (open symbols); although it is out of the range of both experiments, they follow the same "pattern" as the other reflections. The SL reflections could be written in a general form as $\left(2 h \frac{2 k+1}{2}-\delta_{k} \quad 2 l \pm \delta_{l}\right)_{M}$ and $\left(2 h+1 \frac{2 k+1}{2}+\delta_{k} \quad 2 l \pm \delta_{l}\right)_{M}$. If this indexation is correct, 


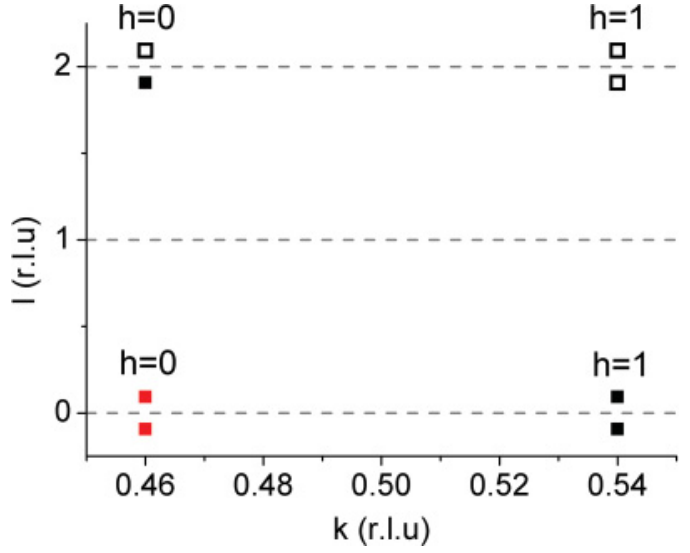

FIG. 9. (Color online) Reciprocal lattice representation of the reflection observed in the present work [filled (red) square] and reflections following the same pattern that fit the neutron powder diffraction study by Nakamura et al. ${ }^{8}$ (filled squares) and extrapolated positions (open squares).

with double peaks at $\pm \delta_{l}$ for all the reflections, this would also mean that the $\mathbf{A}$ and $\mathbf{B}$ reflections are not simply magnetic twins.

Based on this indexation, we have created an approximate magnetic model that reflects the main interactions present in the system. A sketch of this simplified magnetic model is shown in Fig. 10. For this proposed model we make $\delta_{k}=\delta_{l}=0$ in the generalized reflection indexes, which corresponds to $\left(h \frac{2 k+1}{2} 2 l\right)_{M}$. The moments were drawn in the $b c$ plane. The value $k=\frac{2 k+1}{2}$ corresponds to a double-unit cell for the magnetic structure in real space, which implies an antiferromagnetic interaction in the $b$ direction. Having reflections at $2 l$ indicates a periodicity of half of the unit cell along $c$ of the magnetic structure. Since there are four vanadium atoms along the $c$ axis, this indicates that $\mathrm{V} 2$ and $\mathrm{V} 4$ atoms must have a ferromagnetic interaction along $c$. The same is true for V1 and V3, but they must have different magnetic moments than $\mathrm{V} 2$ and V4.

However, from our analysis, it is not possible to predict the interaction between V2 and V4, or between V1 and V3, inside the $a b$ plane. This means that it is not possible to predict the coupling of the vanadium chain from the corners with respect to the center one shown in Fig. 10. The exact direction

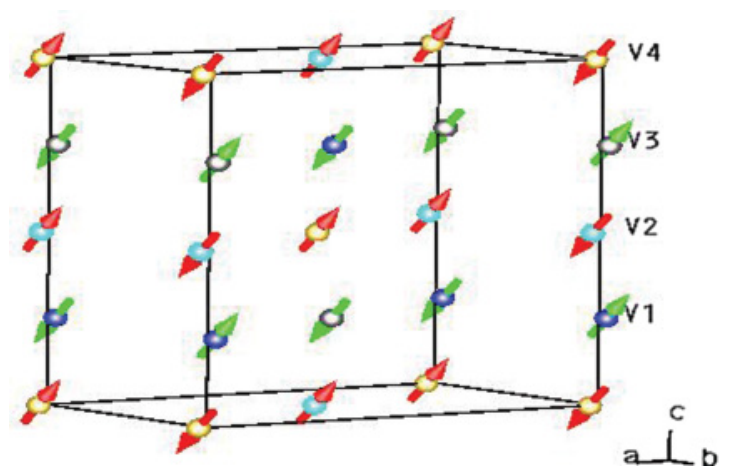

FIG. 10. (Color online) Sketch of a simplified magnetic model of the $\mathrm{V}$ moments in $\mathrm{BaVS}_{3}$. of the magnetic moments and its electronic states, which seem very complicated, will have to await larger, high-quality monodomain crystals being obtained, thus allowing further polarized neutron and/or hard X-ray studies.

The orbitally ordered model proposed by Fagot et al. ${ }^{12}$ (see Fig. 1 there), suggests that V1 and V3, along the $c$ axis, have different orbital types, $A_{1 g}\left(d_{z}^{2}\right)$ and $E_{g}$, respectively. However, different electronic states within the pairs of V1,V3 or V2,V4 would lead to unequal magnetic moments, in both size and direction, and, correspondingly, nonzero intensities for reflections with $l=1$, which have so far not been observed in the neutron experiment.

It is also interesting to compare our results to the theoretical prediction from DFT calculations. ${ }^{22,23}$ A magnetic model has been proposed having antiferromagnetic order (interaction) along the $a$ and $b$ axes, inconsistent with the observed reflection. The proposed pure ferroelectric interaction along the $c$ axis would also allow reflections with $l=2 n+1$. A very recent resonant $\mathrm{x}$-ray study ${ }^{24}$ claims that the magnetic moments lie along the $a$ axis but provides no further information on the coupling along $c$. This interpretation is based on the azimuthal angle dependence of the magnetic reflection using linear polarized light and the assumption of having a single type of magnetic site. Our data, with significant spectral changes for different azimuths, give a clear indication of different electronic and magnetic states, invalidating this assumption. It also shows that the azimuthal angle-dependent intensity ratio taken with different polarizations does depend on the energy. This might be one of the reasons for the relatively poor agreement between the calculated and the observed spectral shapes of the reflection presented in that study. Finally, we note that in these types of studies we are looking at a single reflection (here with $h=0$ in the monoclinic cell). Such single reflections could be insensitive to some of the magnetic components of the magnetic structure due to its particular Miller indexes.

\section{CONCLUSION}

Resonant soft XRD was used to study the low-temperature phase of $\mathrm{BaVS}_{3}\left(T<T_{X} \approx 30 \mathrm{~K}\right)$. The polarization dependence of the signal points to a magnetic origin of the signal, showing that $T_{X}=T_{N}$. The different energy dependencies of the incommensurate reflections with different incident polarizations suggest different electronic contributions to the signal. This confirms that the individual $\mathrm{V}$ ions are in different electronic states. The azimuthal angle dependence measured with circular polarization evidences a magnetic spiral component with vanadium magnetic moments lying in the $b c$ plane. A simplified magnetic structure model, based on the presented X-ray and previous neutron results, ${ }^{8}$ is proposed.

\section{ACKNOWLEDGMENTS}

We benefited from valuable discussions with C. Mazzoli, F. de Bergevin, L. Forró, and A. Gauzzi. We thank the X11MA beamline staff for experimental support. Financial support from the Swiss National Science Foundation and its NCCR MaNEP is gratefully acknowledged. 
${ }^{1}$ M. Imada, A. Fujimori, and Y. Tokura, Rev. Mod. Phys. 70, 1039 (1998).

${ }^{2}$ G. Mihály, I. Kézsmárki, F. Zámborszky, M. Miljak, K. Penc, P. Fazekas, H. Berger, and L. Forró, Phys. Rev. B 61, R7831 (2000).

${ }^{3}$ F. Sayetat, M. Ghedira, J. Chenavas, and M. Marezio, J. Phys. C 15, 1627 (1982).

${ }^{4}$ R. A. Gardner, M. Vlasse, and A. Wold, Acta Crystallogr. Sect. B 25, 781 (1969).

${ }^{5}$ S. Fagot, P. Foury-Leylekian, S. Ravy, J. P. Pouget, and H. Berger, Phys. Rev. Lett. 90, 196401 (2003).

${ }^{6}$ A. Tanaka, J. Magn. Magn. Mater. 310, 898 (2007).

${ }^{7}$ F. Lechermann, S. Biermann, and A. Georges, Phys. Rev. B 76, 085101 (2007).

${ }^{8}$ H. Nakamura, T. Yamasaki, S. Giri, H. Imai, M. Shiga, K. Kojima, M. Nishi, K. Kakurai, and N. Metoki, J. Phys. Soc. Jpn. 69, 2763 (2000).

${ }^{9}$ H. Nakamura, H. Imai, and M. Shiga, Phys. Rev. Lett. 79, 3779 (1997).

${ }^{10}$ Z. V. Popovic, G. Mihaly, I. Kezsmarki, H. Berger, L. Forró, and V. V. Moshchalkov, Phys. Rev. B 65, 132301 (2002).

${ }^{11}$ S. Fagot, P. Foury-Leylekian, S. Ravy, J. P. Pouget, M. Anne, G. Popov, M. V. Lobanov, and W. Greenblatt, Solid State Sci. 7, 718 (2005).

${ }^{12}$ S. Fagot, P. Foury-Leylekian, S. Ravy, J. P. Pouget, E. Lorenzo, Y. Joly, M. Greenblatt, M. V. Lobanov, and G. Popov, Phys. Rev. B 73, 033102 (2006).
${ }^{13}$ U. Staub, M. García-Fernández, Y. Bodenthin, V. Scagnoli, R. A. De Souza, M. Garganourakis, E. Pomjakushina, and K. Conder, Phys. Rev. B 79, 224419 (2009).

${ }^{14}$ A. M. Mulders, S. M. Lawrence, A. J. Princep, U. Staub, Y. Bodenthin, M. García-Fernández, M. Garganourakis, J. Hester, R. Macquart, and C. D. Ling, Phys. Rev. B 81, 092405 (2010).

${ }^{15}$ H. Huriyaki, H. Berger, S. Nishioka, H. Kawakami, K. Hirakawa, and F. Lévy, Synth. Met. 71, 2049 (1995).

${ }^{16}$ U. Staub, V. Scagnoli, A. M. Mulders, K. Katsumata, Z. Honda, H. Grimmer, M. Horisberger, and J. M. Tonnerre, Phys. Rev. B 71, 214421 (2005).

${ }^{17}$ U. Staub, V. Scagnoli, Y. Bodenthin, M. García-Fernández, R. Wetter, A. M. Mulders, H. Grimmer, and M. Horisberger, J. Synchrotron Radiat. 15, 469 (2008).

${ }^{18}$ V. Scagnoli, U. Staub, Y. Bodenthin, R. de Souza, M. GarcíaFernández, M. Garganourakis, A. Boothroyd, D. Prabhakaran, and S. Lovesey, Science 332, 696 (2011).

${ }^{19}$ J. Hill and D. McMorrow, Acta Crystallogr. Sect. A 52, 236 (1996).

${ }^{20}$ K.-Y. Choi, D. Wulferding, H. Berger, and P. Lemmens, Phys. Rev. B 80, 245108 (2009).

${ }^{21}$ J. P. Hannon, G. T. Trammell, M. Blume, and D. Gibbs, Phys. Rev. Lett. 61, 1245 (1988).

${ }^{22}$ X. F. Jiang and G. Y. Guo, Phys. Rev. B 70, 035110 (2004).

${ }^{23}$ A. Sanna, C. Franchini, S. Massidda, and A. Gauzzi, Phys. Rev. B 70, 235102 (2004).

${ }^{24}$ P. Leininger, V. Ilakovac, Y. Joly, E. Schierle, E. Weschke, O. Bunau, H. Berger, J.-P. Pouget, and P. Foury-Leylekian, Phys. Rev. Lett. 106, 167203 (2011). 